\title{
Dementia: time trends and policy responses
}

\section{Authors}

Yu-Tzu Wu ${ }^{1}$, Fiona E Matthews ${ }^{2}$, Carol Brayne ${ }^{1}$

\section{Affiliations}

${ }^{1 .}$ Department of Public Health and Primary Care, Cambridge Institute of Public Health, Forvie Site, University of Cambridge, School of Clinical Medicine, Box 113, Cambridge Biomedical Campus, Cambridge CB2 0SR, United Kingdom

2. MRC Biostatistics Unit, Institute of Public Health, Forvie Site, University of Cambridge, School of Clinical Medicine, Box 113, Cambridge Biomedical Campus, Cambridge CB2 0SR, United Kingdom

\section{Corresponding author}

Carol Brayne MD MSc FRCP FFPH

Professor of Public Health Medicine

Department of Public Health and Primary Care

Cambridge Institute of Public Health, University of Cambridge 
Forvie Site, School of Clinical Medicine, Box 113, Cambridge Biomedical Campus,

Cambridge CB2 0SR, United Kingdom

Email: carol.brayne@medschl.cam.ac.uk

Telephone: +44 1223330321 


\section{Abstract}

In recent years, dementia has been considered a public health priority and become a topic of major political interest. Recent reviews and studies have reported with varying degrees of alarm an impending and existing "dementia epidemic" with increasing predicted trends in prevalence and enormous numbers of people with dementia particularly in low- and middleincome countries (LMICs). However, robust evidence from dementia research in high-income countries suggests stable or decreased prevalence over the last decades. Current evidence is not sufficient to suggest increasing trends of prevalence in LMICs once variation in methodological factors and study populations are taken into account. Changes in diagnostic methods over the last decades substantially influence the identification of dementia cases with systematic difference between the resulting individual prevalence studies. Potential geographical variations at the country level might indicate potential risk factors at population levels or systematic difference in clinical application of dementia diagnosis. Although it is important and necessary to use information from dementia research for evidence-based policymaking, over-interpretation of results without carefully considering underlying factors could exaggerate the findings and influence policy planning in ways which do not serve current and future population best. Planning of dementia policy needs to take full cognisance of the provenance of the data being used and be integrated with policies which optimise health across the lifecourse. 


\section{Keywords}

Dementia; Policy planning; Review

\section{Abbreviations}

CCMD: Chinese Classification of Mental Disease

DSM-III/ -IV/ -V: Diagnostic and Statistical Manual of Mental Disorder Third/ Fourth/ Fifth

Edition

GMS-AGECAT: Geriatric Mental State Examination- Automated Geriatric Examination for

Computer Assisted Taxonomy

ICD-9/ 10: the International Statistical Classification of Diseases 9th / 10th

LMICs: Low- and middle-income countries 


\section{Introduction}

In recent years, dementia has become a public health priority with substantial impact on not only individuals and their families but also health care, economic and welfare systems of whole societies [1]. In 1980s, the governments of developed countries started to express concern about rapid population ageing with dementia and cognitive decline being important causes of disability in later life [2]. To investigate dementia in general populations, several epidemiological studies were conducted in North America and Western Europe between 1980s and 1990s. The findings provided important evidence for health policy planning [3, 4]. Awareness of dementia has increased the need for good data extending from high-income to low- and middle-income countries (LMICs) which now contain large number of ageing population with emerging epidemics of non-communicable diseases. It has also moved from professional to public arenas involving the active campaigns, lobbying of charities and awareness of business opportunities. Dementia has become a topic of major political interest. In the UK, the Prime Minister's Challenge on Dementia commits to delivery of major improvements in dementia care and research by 2015 [5]. The G8 summit held in December 2013 called for international action to address the problem of dementia and brought together policy makers, researchers, pharmaceutical companies and charities from around the world [6].

The summit agreed on an increased spends on dementia research and the development of international collaborations, information and data sharing. 
This surge in interest in ageing populations and health care provisions can and will affect resource availability for dementia research, which should provide better evidence-based strategies for policy planning. Recent reviews and studies have reported with varying degrees of alarm an impending and existing "dementia epidemic" with increasing predicted trends in prevalence and enormous numbers of people with dementia particularly in developing countries [7]. However, these somewhat hyperbolic statements of a worsening situation need to be constantly examined and updated.

\section{Time trends and geographical variations in dementia}

Over the last decades, several reviews or studies have attempted to investigate the epidemiology of dementia in national and international populations with the exploration of temporal and geographical variations of prevalence. Here we summarise existing evidence of epidemiology of dementia worldwide drawing on relevant systematic reviews (or meta-analysis) and recent epidemiological studies designed to compare the changing epidemiology of dementia in the well-defined populations. Consideration of very different economic and social situations across countries exists, thus synthesis of current evidence from dementia research is reported according to country income levels (high vs low- and middle-income countries). 
High-income countries: Western Europe, North America and Japan

Governments of high-income countries have been aware of demographic ageing and potential increase in dementia since 1980s. The EURODEM collaborative study synthesised the results of community-based studies in European countries between 1980s and 1990s [3]. The prevalence of dementia steadily increased with age and showed similar estimates and patterns across different countries. These results were used to predict the number of people with dementia over years and assist in policy making and service provision while few epidemiological surveys were conducted in the post-EURODEM period [8,9]. In the last five years, a small number of new studies which aimed to provide updated estimates and investigate changes in prevalence have repeated earlier methods in the same areas. The findings of these studies suggest stable or reduced prevalence of dementia over the last 20 years [10-12]. The number of people with dementia in European countries is considered to be lower than the estimates in 1990s had predicted given the changes in age structures of these populations.

Similar to Western Europe, the results of early prevalence studies in the US have been used to estimate nationwide prevalence using the projection methodology with different assumptions of demographic ageing [13-16]. In contrast, the two studies comparing repetitive surveys 
across different time periods also reported a stability or decline of the prevalence of dementia and cognitive impairment over time [17-20].

In East Asia, Japan also experienced the pressure of population ageing and conducted early dementia research in 1980s. In contrast to the US and Europe, recent reviews have reported an increasing trend of dementia prevalence in Japan over last decades [21, 22]. However, this finding might be driven by variation of study methods and characteristics of study populations over time [21, 23]. Despite potential bias in study designs and analysis methods, some studies applying the similar study methods in the small areas reported stability of dementia prevalence from 1980s to 1990s but with high prevalence in surveys after 2000 [22, 24, 25]. The prevalence of dementia in Japan could have potentially increased in recent eight to ten years but have been relatively stable before 2000. In South Korea, the estimated prevalence was generally higher than other developed countries since mid-1990s without obvious difference between various diagnostic criteria [26].

Lower- and middle- income countries: global prevalence of dementia and China The 10/66 Dementia Research Group has conducted investigation of dementia prevalence in numerous urban and rural sites in Latin American, India and China using consistent measurement methods [27]. Estimated prevalence of dementia has varied across countries and 
within countries between urban and rural areas. The influence of diagnostic criteria (DSM-IV and 10/66 algorithm) on prevalence estimates has been reported to be substantial in the populations of developing countries [28].

To investigate global burden of dementia and the prevalence in LMICs, the 10/66 Dementia Research Group conducted a systematic review of the worldwide literature from 1980 to 2009 [29]. The results were included in the WHO report of dementia in 2012 [1]. A four-fold variation was found in age-adjusted prevalence of dementia in populations aged 60 and over across regions. Although substantial variation of methodological factors was inevitable, lower estimated prevalence was generally found in African regions while Latin America had particularly high prevalence $[1,30]$. The regions without sufficient prevalence data including Middle East, Eastern and Central Europe, Central Asia and Oceania were based on relevant estimates from the 2005 Delphi Consensus study [29, 31]. The data have been updated in 2013 including newer studies in East Asia and Africa with an estimated prevalence which is higher than the original report [7]. The number of people living with dementia worldwide is estimated to have been nearly 45 million in 2013 and expected to increase to 75 million in 2030 and 135 million in 2050. However, these higher estimates could be substantially driven by recent prevalence studies using newer diagnostic standards based on recent reviews of Chinese studies applied to the massive Chinese population. 
With the largest populations in the world, China started to be concerned about the impact of demographic ageing and dementia more recently. Several reviews have summarised a number of studies since late 1980s [32-35]. The authors report a substantial increase of dementia prevalence in mainland China with a doubling of age-specific prevalence from 1990 to 2010 $[32,34]$. However, this pattern could be largely attributed to variation of study designs and methodological factors as these are significantly related to heterogeneity of prevalence studies in China $[35,36]$. Higher prevalence was found in the recent studies using newer diagnostic criteria (DSM-IV and 10/66 algorithm) than those using older diagnostic criteria (DSM-III, DSM-III-R and CCMD). The increase of dementia prevalence in China is considered therefore to have been amplified by these changes in methodology over time.

In a review of prevalence studies in Brazil from 1990 to 2010, over-estimated prevalence was found in the research with poor quality of study designs such as biased sampling methods, unstandardised measurements and assessors [37]. Methodological variations and quality of study can considerably modify prevalence estimates.

\section{Evidence for time trends and geographical variations}

Instead of expected increasing trends, robust evidence from dementia research in high-income 
countries suggests stable or decreased prevalence over the last decades. The results of recent studies actually suggest that the number of people with dementia in current European populations is stable or lower than the estimates in earlier years. Increasing trends of prevalence have been reported in the low- and middle-income countries mainly based on pooled estimates of the individual studies without taking methodological variations and characteristics of study populations into account. Changes in diagnostic criteria over the last 30 years substantially affect the identification of dementia cases with systematic difference between the prevalence studies using newer and older criteria. Broader definitions of dementia and cognitive decline in recent years could include more suspected and borderline cases with increasing prevalence. Broader definitions of dementia and cognitive decline in recent years seem likely to include more suspected and borderline cases with increasing prevalence particularly in the changes the Diagnostic and Statistical Manual of Mental Disorders, fifth edition (DSM-V). The definition of "neurocognitive disorder", which replaces the term "dementia", could substantially increase the proportion of people diagnosed with mild cognitive decline. New studies using the DSM-V criteria are expected to measure a higher prevalence of dementia and cannot be directly compared with the previous estimates. In developing countries, where dementia research is associated with the development of diagnostic methods and medical services, increasing trends and estimated numbers of people with dementia might be substantially attributed to changes in diagnostic methods rather than 
true increases over time.

Furthermore, societal changes and variations in methodological factors can also importantly moderate the time trends of dementia prevalence. Even though some studies have had to control the influence of various methodological factors, there could be the remaining variation "within diagnostic criteria". Trainings of clinicians and application of diagnostic methods have also changed with time even if subtly. In recent years, the rise of awareness campaigns, changing knowledge and attitude to dementia in professionals and public have made whole societies better prepared to discuss this later life condition. Changes in the social environment might potentially increase the identified number of people with dementia who have previously been considered as a natural stage of ageing and rarely recorded in medical histories. Such variations in diagnostic practice are difficult to measure and the findings of epidemiological studies need to be interpreted very carefully with the consideration of not only design and methods but also social contexts of investigations and health care.

The findings of the 10/66 study indicate geographical variation of dementia prevalence across developing countries and areas. Although the four-fold difference of prevalence at country level was found in the global review, the influence of methodological factors and the development of social environment could potentially moderate the estimates $[1,30]$. Recent 
meta-analysis reviews with taking methodological difference into account suggest an impact on prevalence in different levels of geographical units [34, 38]. Similar to time trends, evidence for geographical variation based on literature reviews has the limitation of not being able to completely adjust for the heterogeneity between individual studies [39]. Definitions of urban/ rural areas and the sizes of city, county and other geographical units can vary considerably across countries and cause difficulty in comparisons.

\section{Challenges and responses}

Since dementia has become a topic of political interest, evidence for time trends and spatial variation has been reported from many epidemiological studies. With this increasing interest and popularity, the conclusions of "enormous numbers", "increasing patterns" and "substantial burden of disease" could be more appealing than any measured reflection on impact of methodologies and conflicting evidence as they can be used by charities, politicians and interested parties (clinicians and specialists) to attract more attention from both public and private sectors including potential investment from pharmaceutical companies and healthcare businesses. Although it is important and necessary to use the information from dementia research for evidence-based policymaking, over-interpretation of results without carefully considering underlying factors could exaggerate the findings and influence policy planning in ways which do not serve current and future populations best. 
Current evidence is not sufficient to suggest increasing trends of prevalence in LMICs taking variations of methodological factors and study populations into account. As health systems in LMICs are still vulnerable with limited resources, over-emphasis on "dementia epidemic" and misleading policy planning might have unintended negative influences on health systems. In the G8 summit, international strategies for dementia were linked to previous experiences of "HIV/ AIDS" and "climate change" [6]. Although the epidemic of HIV/ AIDS in Africa has been addressed successfully, specific funding for HIV/ AIDS projects has driven the priority of health policy and has been reported to seriously interfere with the development of whole societal health systems in these lower income countries [40]. Even though the increasing number of older people is a substantial concern in LMICs, the priority policy should focus on addressing major determinants of health and establishing complete health care systems for basic needs of whole population as they can be beneficial to health of whole societies thus reducing the impact and burden of non-communicable diseases and dementia now and into the future.

Current evidence from high-income countries is actually more optimistic than previous prediction. The reduction of dementia risk in European and the US populations over the last decades is considered to be likely to be associated with earlier life better education, healthy 
lifestyle factors, decreased vascular diseases and other chronic illnesses [20]. Public health policies aimed at whole populations and health care provisions could modify dementia risk in later life over years. This implies that a pure "dementia-orientated policy" could be problematic for effective prevention. Based on the evidence from observational studies, over attention and specific focus on dementia policy and research in secondary (screening) and tertiary (cure and therapy) care might improve individual care provision but have limited influence on population health as key confirmed risk factors and protective features for dementia are related to health conditions in early and middle ages. Policy responses to dementia need not only to consider improvement of care delivery but also integrate with general public health issues (such as smoking, alcohol consumption, physical activity and associations of head injuries) and interventions to reduce impact of other non-communicable diseases.

Although the prevalence of dementia has been investigated in several low- and middle-income countries, there is no available information in the certain regions such as Middle East, Eastern and Central Europe. High prevalence of cardiovascular diseases in these areas could play an important role on modifying the risk of dementia over time with potential geographical variation across countries [41]. To provide robust evidence for time trends in LIMCs, it is important to conduct longitudinal studies with rigorous study designs and quality 
control over time and to investigate the trends in prevalence, trajectories of cognitive function sensitive to the sociocultural environment and changes in risk factors across birth cohorts. Identifying important and specific risk factors in the populations of LIMCs could be an evidence-based and efficient approach for policy planning.

\section{Conclusions}

Interpretation of new results and comparison with earlier studies in the field of dementia need additional consideration of societal changes and research contexts. Stable or reduced prevalence of dementia has been suggested from studies in high-income countries while lack of comparable information over time is a major issue in low- and middle-income countries. Geographical variations at country level might indicate potential risk factors at population levels or systematic difference in clinical application of dementia diagnosis. Changing profiles of potential risk factors in early and middle life could importantly moderate the occurrence of dementia in later life. Planning of dementia policy needs to be integrated with attention to policies which optimise health across the lifecourse. 


\section{Acknowledgements}

There is no specific funding contributing to this study. $\mathrm{Yu}-\mathrm{Tzu} \mathrm{Wu}$ received a $\mathrm{PhD}$ scholarship from the Cambridge Trust, University of Cambridge. Fiona E. Matthews was supported by the Medical Research Council [grant number U105292687]. 


\section{References}

[1] World Health Organisation. Dementia: a public health priority. 2012 http://whqlibdoc.who.int/publications/2012/9789241564458_eng.pdf

[2] Brayne C, Stephan BCM, Matthews FE. A European perspective on population studies of dementia. Alzheimer's \& Dementia. 2011;7(1):3-9.

[3] Hofman A, Rocca WA, Brayne C, et al. The prevalence of dementia in Europe: a collaborative study of 1980-1990 findings. EURODEM Prevalence Research Group. Int J Epidemiol 1991; 20(3): 736-48.

[4] National Institute on Aging. Global health and aging. 2011 http://www.nia.nih.gov/sites/default/files/global_health_and_aging.pdf

[5] UK Department of Health. Prime Minister's challenge on dementia. 2012 https://www.gov.uk/government/uploads/system/uploads/attachment_data/file/215101/dh _133176.pdf

[6] UK government. The G8 dementia summit. 2013. http://dementiachallenge.dh.gov.uk/2013/12/12/g8-dementia-summit-agreements/

[7] Alzheimer's Disease International. The global impact of dementia 2013-2050. 2013. http://www.alz.co.uk/research/GlobalImpactDementia2013.pdf

[8] Misiak B, Cialkowska-Kuzminska M, Frydecka D, Chladzinska-Kiejna S, Kiejna A. 
European studies on the prevalence of dementia in the elderly: time for a step towards a methodological consensus. Int J Geriatr Psychiatry. 2013;28:1211-21.

[9] Lobo A, Launer LJ, Fratiglioni L, Andersen K, Carlo D, Breteler MMB, et al. Prevalence of dementia and major subtypes in Europe: A collaborative study of population-based cohorts. Neurology. 2000;54(11, supp 5):S4-S9.

[10] Qiu C, Strauss E, Bäckman L, Winblad B, Fratiglioni L. Twenty-year changes in dementia occurrence suggest decreasing incidence in central Stockholm, Sweden. Neurology 2013; 80(20):1888-94.

[11] Lobo A, Saz P, Marcos G, et al. Prevalence of dementia in a southern European population in two different time periods: the ZARADEMP Project. Acta Psychiatr Scand 2007; 116(4): 299-307.

[12] Matthews FE, Arthur A, Barnes LE, et al. A two-decade comparison of prevalence of dementia in individuals aged 65 years and older from three geographical areas of England: results of the Cognitive Function and Ageing Study I and II. Lancet 2013; 382(9902):1405-12.

[13] Brookmeyer R, Evans DA, Hebert L, Langa KM, Heeringa SG, Plassman BL, et al. National estimates of the prevalence of Alzheimer's disease in the United States. Alzheimer's \& Dementia. 2011;7(1):61-73.

[14] Hebert LE, Weuve J, Scherr PA, Evans DA. Alzheimer disease in the United States 
(2010-2050) estimated using the 2010 census. Neurology. 2013.

[15] Evans DA. Estimated prevalence of Alzheimer's Disease in the United States. Millbank Q. 1990; 68:267-189

[16] Alzheimer's Association. 2014 Alzheimer's Disease Facts and Figures. http://www.alz.org/downloads/facts_figures_2014.pdf

[17] Rocca WA, Petersen RC, Knopman DS, Hebert LE, Evans DA, Hall KS, et al. Trends in the incidence and prevalence of Alzheimer's disease, dementia, and cognitive impairment in the United States. Alzheimer's \& Dementia. 2011;7(1):80-93.

[18] Manton KC, Gu XL, Ukraintseva SV. Declining prevalence of dementia in the U.S. elderly population. Adv Gerontol 2005;16: 30-7.

[19] Langa KM, Larson EB, Karlawish JH, et al. Trends in the prevalence and mortality of cognitive impairment in the United States: is there evidence of a compression of cognitive morbidity? Alzheimer's \& Dementia 2008;4: 134-44.

[20] Larson EB, Yaffe K, \& Langa KM. New Insights into the Dementia Epidemic. New England Journal of Medicine 2013; 369(24): 2275-77.

[21] Dodge HH, Buracchio TJ, Fisher GG, Kiyohara Y, Meguro K, Tanizaki Y, et al. Trends in the Prevalence of Dementia in Japan. International journal of Alzheimer's disease. 2012;2012:11.

[22] Okamura H, Ishii S, Ishii T, Eboshida A. Prevalence of Dementia in Japan: A Systematic 
Review. Dementia and geriatric cognitive disorders. 2013;36(1-2):111-8.

[23] Wada-Isoe K, Uemura Y, Nakashita S, Yamawaki M, Tanaka K, Yamamoto M, et al. Prevalence of Dementia and Mild Cognitive Impairment in the Rural Island Town of Ama-cho, Japan. Dementia and geriatric cognitive disorders extra. 2012;2(1):190-9.

[24] Sekita A, Ninomiya T, Tanizaki Y, et al. Trends in prevalence of Alzheimer's disease and vascular dementia in a Japanese community: the Hisayama Study. Acta Psychiatr Scand $2010 ; 122: 319-25$

[25] Wakutani Y, Kusumi M, Wada K, Kawashima M, Ishizaki K, Mori M, et al. Longitudinal changes in the prevalence of dementia in a Japanese rural area. Psychogeriatrics : the official journal of the Japanese Psychogeriatric Society. 2007;7(4):150-4.

[26] Cho MJ, Lee JY, Kim B-S, Lee HW, Sohn JH. Prevalence of the Major Mental Disorders among the Korean Elderly. J Korean Med Sci. 2011;26(1):1-10.

[27] de Rodriguez J, Ferri CP, Acosta D, Guerra M, Huang Y, Jacob KS, et al. Prevalence of dementia in Latin America, India, and China: a population-based cross-sectional survey. Lancet. 2008;372(9):464-74.

[28] Prince MJ, de Rodriguez JL, Noriega L, Lopez A, Acosta D, Albanese E, et al. The 10/66 Dementia Research Group's fully operationalised DSM-IV dementia computerized diagnostic algorithm, compared with the 10/66 dementia algorithm and a clinician diagnosis: a population validation study. BMC Public Health. 2008;8:219. 
[29] Prince M, Bryce R, Albanese E, Wimo A, Ribeiro W, Ferri CP. The global prevalence of dementia: A systematic review and metaanalysis. Alzheimer's \& Dementia 2013;9(63-75).

[30] Kalaria RN, Maestre GE, Arizaga R, Friedland RP, Galasko D, Hall K, et al. Alzheimer's disease and vascular dementia in developing countries: prevalence, management, and risk factors. The Lancet Neurology. 2008;7(9):812-26.

[31] Ferri C, Prince M, Brayne C, Brodaty H, Fratiglioni L, Ganguli M, et al. Global prevalence of dementia: a Delphi consensus study. Lancet. 2005;366(9503):2112-7.

[32] Chan KY, Wang W, Wu JJ, et al. Epidemiology of Alzheimer's disease and other forms of dementia in China, 1990-2010: a systematic review and analysis. Lancet 2013; 381(9882):2016-23.

[33] Dong M, Peng B, Lin XT, Zhao J, Zhou YR, Wang RH. The prevalence of dementia in the People's Republic of China: a systematic analysis of 1980-2004 studies. Age \& Ageing. 2007;36:619-24.

[34] Zhang Y, Xu Y, Nie H, Lie T, Wu Y, Zhang L, Zhang M. Prevalence of dementia and major dementia subtypes in the Chinese populations: A meta-analysis of dementia prevalence surveys, 1980-2010. J Clin Neurosci. 2012; http://dx.doi.org/10.1016/j.jocn.2012.01.029.

[35] Wu Y-T, Lee H-y, Norton S, et al. Prevalence studies of dementia in mainland China, 
Hong Kong and Taiwan: a systematic review and meta-analysis. PLoS ONE 2013;

8:e66252.doi:10.1371/journal.pone.0066252

[36] Wu Y-T, Lee H-y, Norton S, et al. Period, birth cohort and prevalence of dementia in mainland China, Hong Kong and Taiwan: a meta-analysis. International Journal of Geriatric Psychiatry 2014; doi: 10.1002/gps.4148

[37] Fagundes SD, Silva MT, Thees MFRS, Pereira MG. Prevalence of dementia among elderly Brazilians: a systematic review. Sao Paulo Med J. 2011;129(1):46-50.

[38] Russ TC, Batty GD, Hearnshaw GF, Fenton C, Starr JM. Geographical variation in dementia: systematic review with meta-analysis. International Journal of Epidemiology 2012;41(4):1012-32.

[39] de Pedro-Cuesta J, Virues-Ortega J, Vega S, Seijo-Martinez M, Saz P, Rodriguez F, et al. Prevalence of dementia and major dementia subtypes in Spanish populations: A reanalysis of dementia prevalence surveys, 1990-2008. BMC neurology. 2009;9(1):55.

[40] Maeseneer JD, Weel Cv, Egilman D, Mfenyana K, Kaufman A, Sewankambo N, et al. Funding for primary health care in developing countries. BMJ. 2008;336(7643):518-9.

[41] Pająk A, Kozela M. Cardiovascular Disease in Central and East Europe. Public Health Reviews. 2012;33(2):416-35. 